\title{
Myoepithelial differentiation subsequent to giant basal cell carcinoma in the left face
}

\author{
Yu-Kun Zhao', Yan-Bin Chen², Li-Li Chen ${ }^{3}$, Yang Li ${ }^{3}$ Xiang Li², Di-Qing Luo ${ }^{1}$
}

\begin{abstract}
'Department of Dermatology, The East Division of The First Affiliated Hospital, Sun Yat-sen University, Guangzhou, China 2Department of Stomatology, The East Division of The First Affiliated Hospital, Sun Yat-sen University, Guangzhou, China ${ }^{3}$ Department of Pathology, The First Affiliated Hospital, Sun Yat-sen University, Guangzhou, China
\end{abstract}

Adv Dermatol Allergol 2022; XXXIX (1): 223-225

DOI: https://doi.org/10.5114/ada.2022.113610

A 71-year-old man presented with 11 years of asymptomatic facial ulcer that was a tiny papule initially, then increased in size slowly with central erosion over the years. A few months before the presentation, a subsequent papule in the previous lesion presented which was asymptomatic and enlarged in size gradually. His medical and family histories were unremarkable. Cutaneous examination revealed that an ulcerated plaque of about $10 \mathrm{~cm} \times 6 \mathrm{~cm}$ in across located on the left cheek, associated with irregularly raised circular hyperpigmented papules around and scars on the centre, and a red erosive nodule-plaque about $2.5 \mathrm{~cm}$ in diameter localized on the left lower quadrant of the ulcer near the middle of mandible (Figure 1). Computed tomography scan showed no lymphatic metastasis. The lesion was completely excised without recurrence in a 9-year follow-up.

The histopathology showed that the sample from the primary ulcer was composed of multinodular tumour nests of small basaloid cells with peripheral palisading, and artifactual retraction between tumour cells, and stroma also presented commonly (Figure $2 \mathrm{~A}$ ). The tumour cells had enlarged hyperchromatic nuclei with inconspicuous nucleoli and scant amounts of eosinophilic cytoplasm, with numerous mitotic and apoptotic figures. The section from the subsequent plaque revealed 2 types of tumour cells including basaloid cells mentioned above and spindle cells; the spindle cells distributed immediately adjacent to typical BCC cells and abounded with eosinophilic cytoplasm in nest arrangement (Figures 2 B, C). Both tumour cells infiltrated into the superficial dermis (Figure 2 C), and more spindle cells presented in the centre of the subsequent plaque. However, no typical signet ring cells were detected. Immunohistochemical staining revealed that the basaloid cells were diffusely positive for P63, CK and CK5/6, focally positive for calponin, and weakly reactions for actine and CD10. The spindle cells were strongly positive for actine (Figure $2 \mathrm{C}$ ), calponin (Figure 2 D), CD10, P63, CK and CK5/6, and focally positive for $\mathrm{S100}$. Both kinds of tumour cells were negative reactions for ESA, MSA, GFAP, H-caldesmon, CD34 as well as Ber-EP4. Subcutaneous tissue was not involved. The annular lesion was finally diagnosed as basal cell carci-

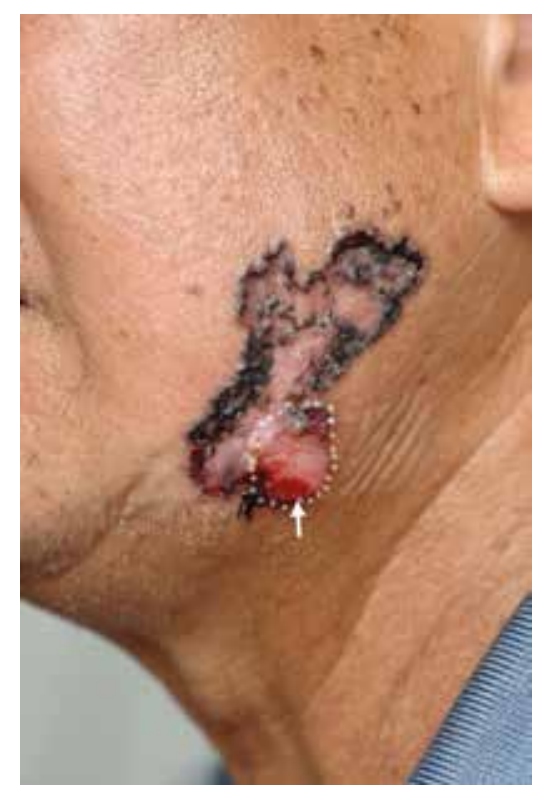

Figure 1. Ulcerated lesion with a central depression and rolled border and raised circular hyperpigmented papules around on the left cheek, and an erosive nodule-plaque about $2.5 \mathrm{~cm}$ in diameter on the left lower quadrant of the ulcer near the middle of mandible (the circular)

Address for correspondence: Prof. Xiang Li, Department of Stomatology, The East Division of The First Affiliated Hospital, Sun Yat-sen University, Guangzhou 510700, phone: +86 20 82379472; fax: +86 20 82398840, e-mail: hpyylx@163.com; Prof. Di-Qing Luo, Department of Dermatology, The East Division of The First Affiliated Hospital, Sun Yat-sen University, 183 Huangpu Rd. E., Guangzhou 510700, China, phone: +86 20 82493439, fax: +86 20 82398840, e-mail: luodq@mail.sysu.edu.cn Received: 5.04.2020, accepted: 28.06.2020. 

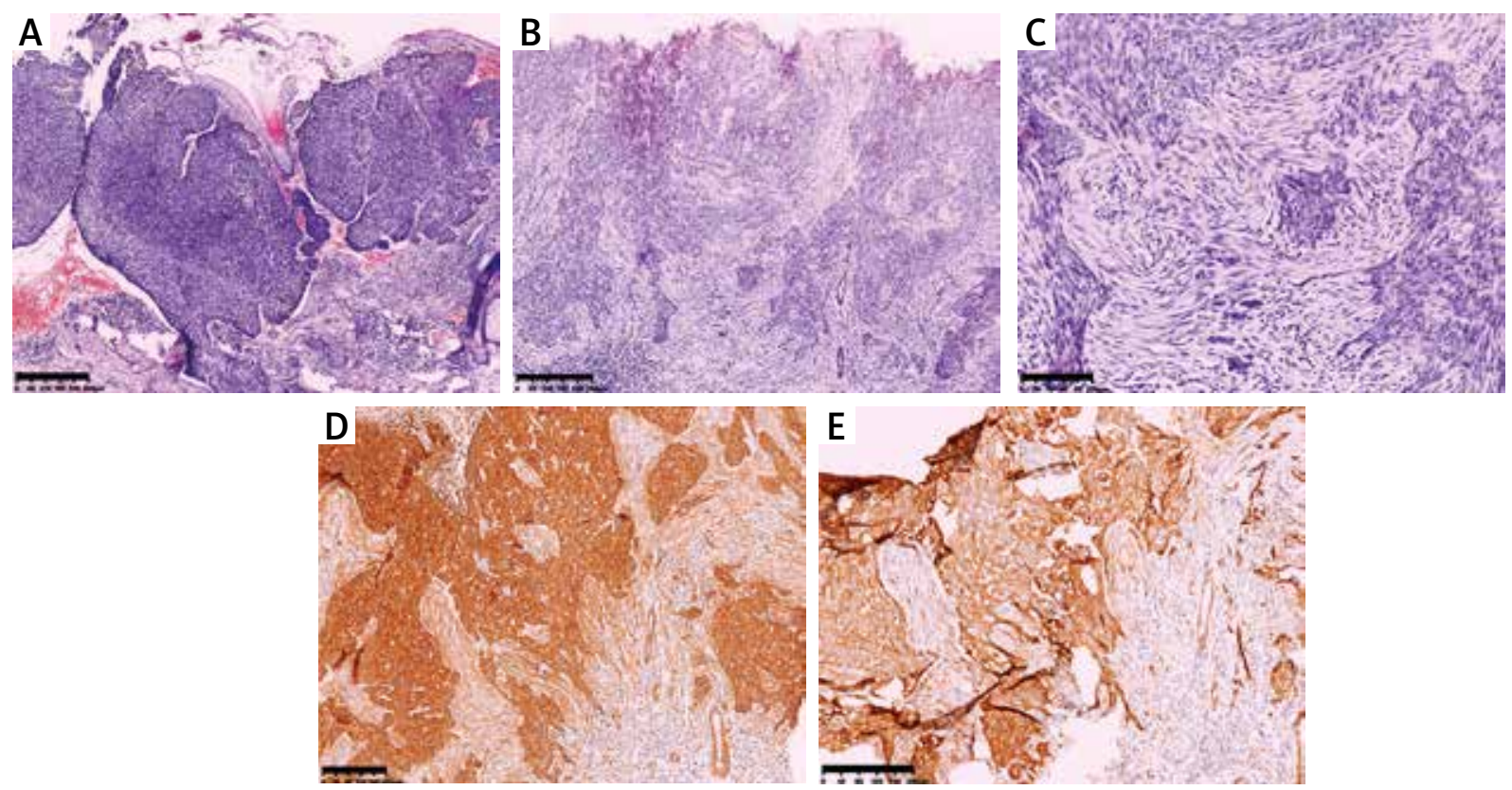

Figure 2. The histopathology revealed that tissue from the ulcer showed multinodular tumour nests of typical BCC in the dermis (A - Haematoxylin-eosin staining), while that from the plaque was composed of two different cells including basaloid cells and spindle cells in the dermis (B, C - Haematoxylin-eosin staining, $\mathbf{C}$ is the magnification of $\mathbf{B}$ ), the spindle cells distributed immediately adjacent to typical BCC cells and abounded with eosinophilic cytoplasm (C). A sample from the plaque showed that both typical basal cell carcinoma component and the spindle cells were positive for actine (D) and calponin (E) (the bars for A-E are 300, 300, 100, 200 and $200 \mu \mathrm{m}$, respectively)

noma (BCC) and the subsequent plaque was BCC with myoepithelial differentiation (MED).

In the skin, myoepithelial cells are generally located at the periphery of eccrine and apocrine glands near to the basement membrane and may be spindle shaped, epithelioid, plasmacytoid, hyaline, or clear [1]. In rare instances, the cells may present the morphology of a signet ring cell $[1,2]$. It is also well known that BCC can differentiate diversely to other cells or organs, such as squamous cell, sweat duct, sebaceous gland, even with MED [1-5]. Histopathologically and immunohistochemically, the cells of BCC with MED consist of 2 different components: typical BCC or mixed basal cells, and plasmacytoid-like cells as well as spindle cells with hyaline inclusions and eosinophilic cytoplasm and compressed nuclei resembling that of signet ring cells [1-4]. In rare cases, the signet ring cell proliferation was focal [2]. Both spindle and plasmacytoid-like cells share the immunohistochemical patterns of myoepithelial cells, to varying degrees, including positive stain of keratin, smooth muscle actin, glial fibrillary acid protein, S100 as well as vimentin respectively, but might be negative for carcinoembryonic antigen and des$\min [1,5]$. As the spindle cells of the present patient were distributed immediately adjacent to typical BCC cells and showed positive reactions for calponin and actine associated with increased numbers, suggesting that such cells are myoepithelial differentiation rather than normal myoepithelial cells and mesenchymal as well as desmoplas- tic stromal response to BCC. Based on histopathological and immunohistochemical features, the present patient could be diagnosed with BCC with MED rather than coexistence of those two respective entities. We speculated that the patient had typical BCC initially, then the tumour cells occurred/showed myoepithelial differentiation in subsequent, although a possible coexistence of both cells could not be excluded absolutely. Interestingly, no typical signet ring cells were detected for the present patient.

Up to date, only 17 cases of BCC with MED, including the present, have been reported, including 12 males and 5 females, with age range of 43 to 83 years and median age more than 67 years, the lesional sizes range from $3 \mathrm{~mm} \times 3 \mathrm{~mm}$ to $35 \mathrm{~mm} \times 28 \mathrm{~mm} \times 15 \mathrm{~mm}$, and 10 cases were less than $10 \mathrm{~mm} \times 10 \mathrm{~mm}$ [1-6]. To our knowledge, the plaque of the present case was the largest. Women were younger than men when their diagnoses were made $[1,4-6]$. All the reported cases involved the face alone [1-6], however, the reason for facial predilection remains unknown.

BCC with MED should be distinguished from sarcomatous component of a biphasic neoplasm, desmoplastic stromal response to the BCC, myoepithelioma, myoepithelial carcinoma, mixed cutaneous benign tumours of either apocrine or eccrine origin, and plasmacytoid variant squamous cell carcinoma, some kinds of metastatic carcinoma from the gastrointestinal tract and from the breast, T-cell lymphoma, etc. [1, 5, 7]. Based on their his- 
topathological and immunohistochemical alterations, making a correct diagnosis is not difficult. Completely lesional excision is an optimal option for BCC with MED $[1,4-6]$.

\section{Acknowledgments}

The authors sincerely thank the patient participating the present work.

Yu-Kun Zhao and Yan-Bin Chen contributed equally to the present work.

\section{Conflict of interest}

The authors declare no conflict of interest.

\section{References}

1. Zheng S, Guo Y, Mones JM. Basal cell carcinoma with myoepithelial differentiation. Am J Dermatopathol 2011; 33: 863-6.

2. Suster S, Ramon Y, Cajal S. Myoepithelial differentiation in basal cell carcinoma. Am J Dermatopathol 1991; 13: 350-7.

3. Chang YT, Liu HN, Wong CK. Penile basal cell carcinoma with eccrine differentiation. Clin Exp Dermatol 1995; 20: 487-9.

4. Kim YC, Vandersteen DP, Chung YJ, et al. Signet ring cell basal cell carcinoma: a basal cell carcinoma with myoepithelial differentiation. Am J Dermatopathol 2001; 23: 525-9.

5. Cohen PR. Basal cell carcinoma with myoepithelial differentiation: case report and literature review. Cureus 2018; 10: e2081.

6. Huang Y, Quan J, Hu H, et al. Basal cell carcinoma with myoepithelial differentiation. Am J Dermatopathol 2012; 34: 850-3.

7. Hornick JL, Fletcher CD. Cutaneous myoepithelioma: a clinicopathologic and immunohistochemical study of 14 cases. Hum Pathol 2004; 35: 14-24. 